\title{
A protein interaction map identifies existing drugs targeting SARS-CoV-2
}

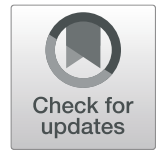

\author{
Claudia Cava $^{1 *}$ (D), Gloria Bertoli ${ }^{1}$ and Isabella Castiglioni ${ }^{2}$
}

\begin{abstract}
Background: Severe acute respiratory syndrome coronavirus (SARS-CoV-2), an emerging Betacoronavirus, is the causative agent of COVID-19. Angiotensin converting enzyme 2 (ACE2), being the main cell receptor of SARS-CoV-2, plays a role in the entry of the virus into the cell. Currently, there are neither specific antiviral drugs for the treatment or preventive drugs such as vaccines.

Methods: We proposed a bioinformatics analysis to test in silico existing drugs as a fast way to identify an efficient therapy. We performed a differential expression analysis in order to identify differentially expressed genes in COVID19 patients correlated with ACE-2 and we explored their direct relations with a network approach integrating also drug-gene interactions. The drugs with a central role in the network were also investigated with a molecular docking analysis.
\end{abstract}

Results: We found 825 differentially expressed genes correlated with ACE2. The protein-protein interactions among differentially expressed genes identified a network of 474 genes and 1130 interactions.

Conclusions: The integration of drug-gene interactions in the network and molecular docking analysis allows us to obtain several drugs with antiviral activity that, alone or in combination with other treatment options, could be considered as therapeutic approaches against COVID-19.

Keywords: COVID-19, SARS-CoV-2, Drug, Network, In silico analysis, Molecular docking

\section{Background}

Coronaviruses are a group of single-stranded RNA viruses with a wide range of vertebrate hosts.

Coronavirus infection in humans is characterized by a wide spectrum of clinical manifestations ranging from mild influenza symptoms to acute respiratory syndrome with damages in lung function, pneumonia, arrhythmia, or death [1].

Betacoronavirus $(\beta-\mathrm{CoV})$ is the second of four genera of coronaviruses: alpha, beta, gamma and delta. Over the past two decades, three highly pathogenic human betacoronaviruses have spread from animals to humans: Severe Acute Respiratory Syndrome Coronavirus (SARS-

\footnotetext{
* Correspondence: claudia.cava@ibfm.cnr.it

${ }^{1}$ Institute of Molecular Bioimaging and Physiology, National Research Council (IBFM-CNR), Via F. Cervi 93, 20090 Segrate-Milan, Milan, Italy

Full list of author information is available at the end of the article
}

$\mathrm{CoV}$ ) in 2002-2003, MERS-CoV in 2012 and SARS$\mathrm{CoV}-2$ in 2019. Compared to SARS-CoV or MERS-CoV, SARS-CoV-2 spreads more rapidly making it difficult to control [1].

Recently, a first model of infection has been proposed [2] in which, after an initial phase of viral exposure, the local innate immune response generates natural IgA and IgM antibodies. In this first stage (5-6 days after infection) patients show their first symptoms limited to upper airways (cough, sore throat) with fever, fatigue and muscle ache. If SARS-CoV-2 (the virus of COVID-19) blocks the innate immunity, it spreads initially from the upper airways to the alveoli, causing pneumonia and dyspnea, and releasing high amounts of viral particles. While many subjects remain asymptomatic or with mild symptoms, in other individuals the response of adaptive immunity, releasing IgM and IgG antibodies, causes

(c) The Author(s). 2020 Open Access This article is licensed under a Creative Commons Attribution 4.0 International License, which permits use, sharing, adaptation, distribution and reproduction in any medium or format, as long as you give appropriate credit to the original author(s) and the source, provide a link to the Creative Commons licence, and indicate if changes were made. The images or other third party material in this article are included in the article's Creative Commons licence, unless indicated otherwise in a credit line to the material. If material is not included in the article's Creative Commons licence and your intended use is not permitted by statutory regulation or exceeds the permitted use, you will need to obtain permission directly from the copyright holder. To view a copy of this licence, visit http://creativecommons.org/licenses/by/4.0/. The Creative Commons Public Domain Dedication waiver (http://creativecommons.org/publicdomain/zero/1.0/) applies to the data made available in this article, unless otherwise stated in a credit line to the data. 
severe symptoms such as acute respiratory distress syndrome (ARDS), due to spreading of inflammation, often leading to complications (dyspnea, respiratory failure, fulminant myocarditis) that require the use of intensive therapy. In the last phase, COVID-19 could cause death due to ARDS, acute cardiac and kidney injury, sepsis, and secondary infections. High mortality has been observed in patients with older age or comorbidities (hypertension, diabetes, cardiovascular disease, chronic lung disease and cancer).

Several studies reported that the first sites of infection seem to hit pneumocytes and enterocytes of small intestine [3]. The molecular mechanisms of SARS-CoV-2 entry, as suggested also for SARS-CoV [4], is mediated by the binding of the viral spike glycoprotein to the angiotensin-converting-enzyme-2 (ACE2) receptor, in collaboration with a serine protease, TGRBSS2. This allows the viral capsid fusion with the cell membrane [4].

SARS-CoV-2 is a new virus for our immune system. The IgG antibodies produced by other $\beta-\mathrm{CoV}$ do not recognise this SARS-CoV-2, making this virus currently invincible. A possibility would be the use of immunoglobulin collected from immunized COVID-19 patients to be injected in patients suffering from COVID-19 [5]. In silico approaches on the study of the binding of existing antiviral drugs on cellular receptors to block viral protein entrance could be a fast and low-cost method to test a large amount of possible therapies.

Furthermore, immunotherapy approach combined with antiviral drugs detected by in silico analyses may be a stronger treatment until definitive options such as vaccines are available.

In this work we propose in silico study to identify genes deregulated in COVID-19 positive patients correlated with ACE2. Then, we performed a network analysis based on protein-protein interactions with the aim of identifying existing drugs targeting SARS-CoV-2 deregulated genes, to be proposed as candidate drugs for COVID-19, alone or in combination with other therapeutic strategies. The protein interactions that emerge from our study could help to decipher their mechanisms of action with respect complex biological processes at the basis of COVID-19 infection and progression. The drugs obtained were also investigated through docking simulations.

\section{Methods}

\section{Gene expression datasets}

We collected the gene expression levels of lung tissues infected by SARS-CoV-2 from the Gene Expression Omnibus (GEO) database. Specifically, we selected from GSE147507 2 lung biopsies from postmortem COVID19 positive male patients and 2 lung biopsies from COVID-19 negative male controls.
Furthermore, from the Genotype-Tissue Expression (GTEx) project we collected gene expression levels of lung tissues from healthy subjects $(320$ healthy volunteers).

\section{Differential expression, pathway and network analysis}

We normalized and filtered RNA-seq raw counts using the reference of hg19, following the pipeline of the R/ Bioconductor package TCGAbiolinks [6]. In particular, we used the function TCGAanalyze_Normalization implementing the EDASeq protocol [7], to perform within-lane and between-lane normalization. We adjusted the data for differences in gene length and distributional differences between samples [7].

Differential expression analysis between lung biopsies from postmortem COVID-19 positive male patients and negative male controls was performed on GSE147507 gene expression levels with the R-package TCGABiolinks [6]. Specifically, we used a generalized linear model which is similar to linear model, but it assumes the negative binomial distribution of RNA-Seq counts. P-values, generated from the differential expression analysis, were corrected using the Benjamini-Hochberg procedure for multiple-testing correction [6]. We defined the differentially expressed genes if FDR $<0.01$ and $\mid \log$. FC $\mid>1$.

Pathway enrichment analysis was performed with the R-package clusterProfiler [8]. In particular, we performed an enrichment test for KEGG pathways based on hypergeometric distribution [9]. We identified biological functions that are over-represented in the list of differentially expressed genes [10].

We generated a protein-protein interaction network considering the direct connections among differentially expressed genes in COVID-19 using SpidermiR [11] and we selected the network with the most connected nodes. We calculated for each node the degree centrality (d.c.) that indicates how many neighbours a node has. This index is usually used to identify the nodes that have a central role in the network [11].

Drug-gene interactions were derived by the package rDGIdb [12].

\section{Correlation}

A correlation analysis was performed between ACE2 and the other genes in GTEx data (lung tissues from normal volunteers) to obtain the genes co-expressed with ACE2. The corresponding $p$-values of the Pearson's correlation were considered and only ACE2 and genes significantly correlated ( $p$-value $<0.001$ ) were used for the subsequent analysis.

\section{Community detection}

We implemented the fast greedy modularity optimization algorithm for finding community structure of our network 
[13]. Community detection algorithms refer to the procedures for finding groups of connected nodes in a network based on their structural properties [13].

Indeed, a network can be divided into communities or clusters and each community is characterized by many edges within communities and only a few edges between communities.

\section{Molecular modelling: ligands and protein structures preparation}

Docking study explored the binding mode of reported drugs on the 3D model of protease of SARS-CoV-2.

Based on the above analysis, the structural coordinates of potential drugs (file SDF) were downloaded from PubChem [14]. DiscoveryStudio Visualizer was used to generate protein data bank (PDB) format of the ligand from its 3D structure (file SDF) [15] downloaded by PubChem.

Ligand preparation and molecular modelling are performed using AUTODOCK 1.5.6 [15] following the procedure in [16].

After, the ligand is loaded, AUTODOCK automatically prepares it for molecular docking. The software computes Gasteiger charges and if the charges are all zero, it will add charges [16, 17].

Crystal structures of SARS-CoV-2 main proteases (PDB IDs: 5R7Y, 5R7Z, 5R80, 5R81 and 5R82) were obtained from Protein Data Bank. Polar hydrogen atoms were added, and water molecules were removed to the SARS-CoV-2 model.

The macromolecules (main protease) and ligands were saved in pdbqt format to be used for docking analysis. The grid parameters in the $\mathrm{x}, \mathrm{y}$ and $\mathrm{z}$ dimensions were set to $90,90,90$. Autodock uses the Lamarckian genetic algorithm to search for the ligand exposure with the highest binding affinity. Default settings were used for other parameters following the docking protocol reported in [16].

Binding energies between ligand and main protease (docking scores) are reported in $\mathrm{kcal} / \mathrm{mol}$. High negative values suggest a better binding $[16,17]$.

Protein-Ligand Interaction Profiler was used to visualize the binding interactions of the selected drugs with 3D model of protease of COVID-19 [18].

\section{Results}

Differential expression and pathway analysis reveal novel aspects of SARS-CoV-2 biology

Our study revealed 1269 differentially expressed genes between lung biopsies from postmortem COVID-19 positive patients and lung biopsies from negative controls (Supplementary 1). Volcano plot was designed to visualize the distribution of each gene related to the fold change and $p$-value (Fig. 1a).
We found 22 pathways enriched with 1269 differentially expressed genes as reported in Fig. 1b. The top differentially expressed mRNAs were associated with Alzheimer's disease, human T-cell leukemia virus 1 infection, Shigellosis, hepatocellular carcinoma, viral carcinogenesis, apoptosis, non-alcoholic fatty liver disease (NAFLD), mTOR signaling, cellular senescence, protein processing in endoplasmic reticulum and cell cycle.

\section{Differentially expressed genes in COVID-19 are correlated with ACE2}

From the correlation analysis in GTEx data, we obtained 11,011 genes that correlated with ACE2. We selected differentially expressed genes in COVID-19 that also correlated with ACE2 in GTEX data: $65 \%$ of differentially expressed genes (825 genes out of 1269) were found.

\section{Protein-protein interaction reveals existing drugs targeting SARS-CoV-2}

We studied the interactions among differentially expressed genes correlated with ACE2. From the analysis of protein-protein interaction network, we obtained a network of 474 genes and 1130 interactions. We queried DGIdb and we obtained 714 drugs that interact with 119 of 474 genes involving 950 interactions.

We integrated protein-protein interactions' information with the drug-gene interactions obtaining a network of 1188 nodes (including 474 genes and 714 drugs) and 2080 interactions. The overview of such network is shown in Fig. 2a.

The drugs with the highest degree centrality (d.c.) are: CHEMBL1161866 (d.c. = 11), bortezomib (d.c. =6), puromycin (d.c. $=6)$, metformin hydrochloride $($ d.c. $=6)$, NV-128 (d.c.=6), ME-344 (d.c.=6), methylene blue $($ d.c. $=5)$, anisomycin $($ d.c. $=5)$ and everolimus (d.c. $=5)$ (Fig. 2b).

The d.c. of the drugs in the network indicates the number of interacting genes. Higher d.c. drugs suggest their central role in the network and the ability to regulate a major number of target genes.

The proteins with the highest d.c. are: ERBB2 (d.c. = 146), $\operatorname{APP}($ d.c. $=143), \operatorname{SUMO} 2$ (d.c. $=106)$, CUL3 (d.c. $=$ 89), CA2 (d.c. $=72)$, GSK3B (d.c. $=61)$, HSPA4 (d.c. $=60)$, NRAS (d.c. $=53)$ and MTOR (d.c. $=50)($ Fig. 2c).

Moreover, communities detection algorithm identified 19 communities (Fig. 2d), the biggest communities consisting of 239, 175 and 174 nodes (see Fig. 2d).

In the first community we found 3 genes with the highest d.c.: CUL3 (d.c. =54), SUMO2 (d.c. =53), and APP (d.c. =41). The drugs with key roles in the network are 7: bortezomib, puromycin, ixazomib citrate, carfilzomib, oprozomib, methylene blue and anisomycin. The drugs with the major number of drug targets in the 


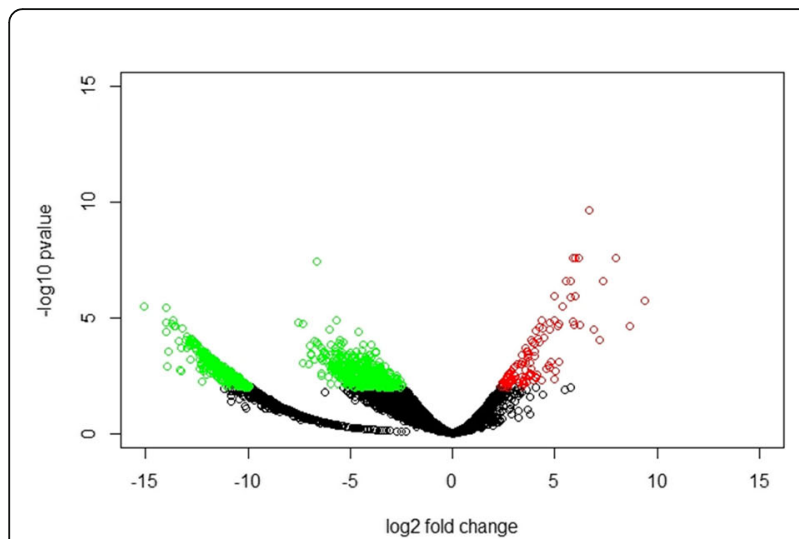

A)

Fig. 1 a Volcano plot for differential gene expression. The dots represent the genes that are related to $p$-value versus fold change. Red dots are genes that are significantly up-regulated in Covid-19, and green dots are genes significantly down-regulated in Covid-19. b Top enriched KEGG pathways.

The size of the circles represents the number of differentially expressed gene in the pathway. The color intensity of the circle represents the p-value network are: puromycin, methylene blue and anisomycin. They interact with 5 proteins: RPL11, RPL15, RPL26L1, RPL37 and RPL8 (Fig. 3a).

In the second community we found 3 genes with the highest d.c.: ERBB2 (d.c. = 25), NRAS (d.c. = 22) and RB1 $($ d.c. $=10)$. The drugs involved in this community are 27 : metformin, abemaciclib, ribociclib, palbociclib, pictilisib, fluorouracil, trametinib, vorinostat, sirolimus, entinostat, everolimus, cetuximab, crizotinib, sorafenib, afatinib, ethyl nitrosourea, gefitinib, osimertinib, mk-2206, buparlisib, gedatolisib, bgb-283, panitumumab, selumetinib, chembl1229592, vemurafenib and dabrafenib. The drug

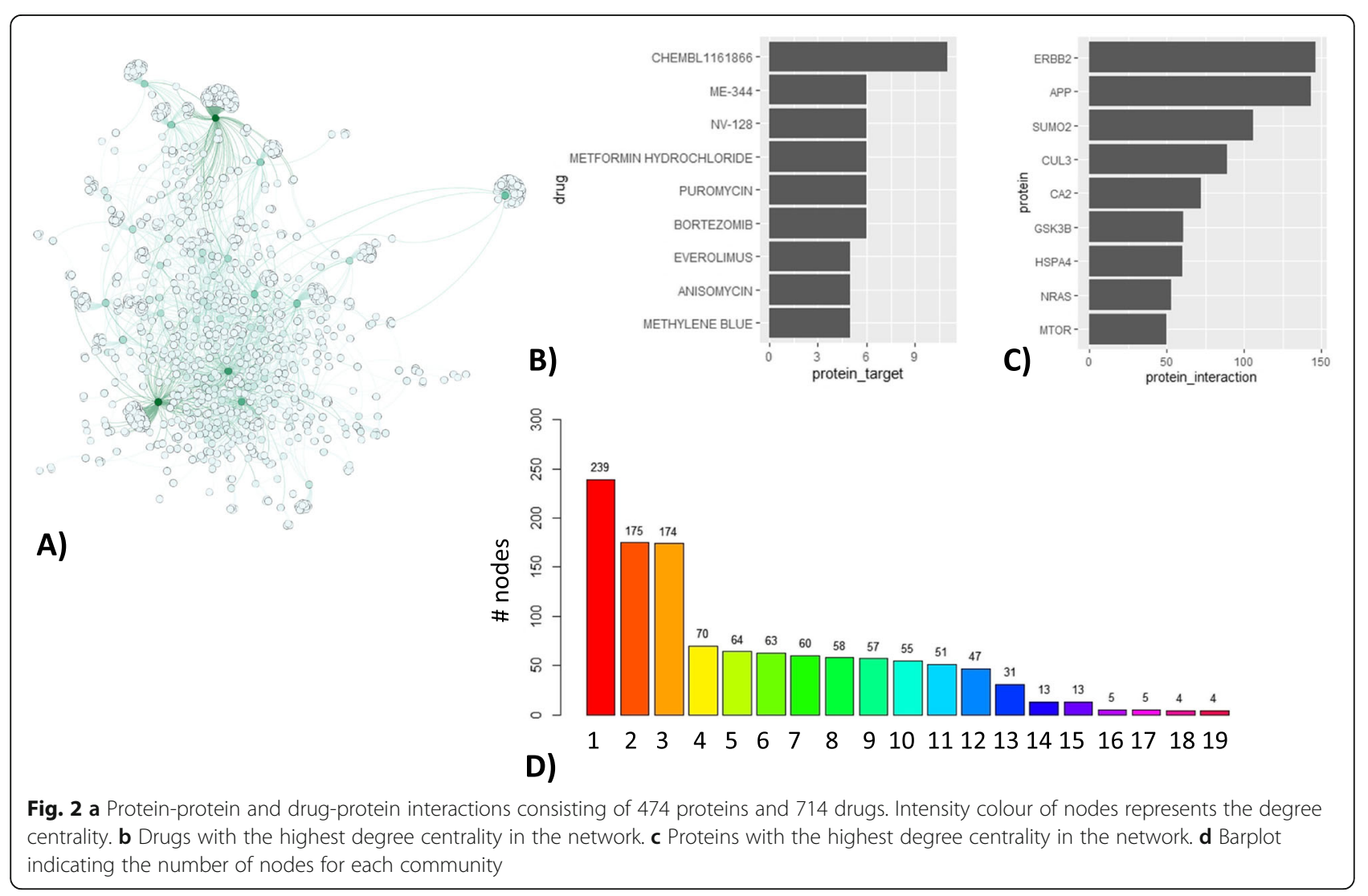




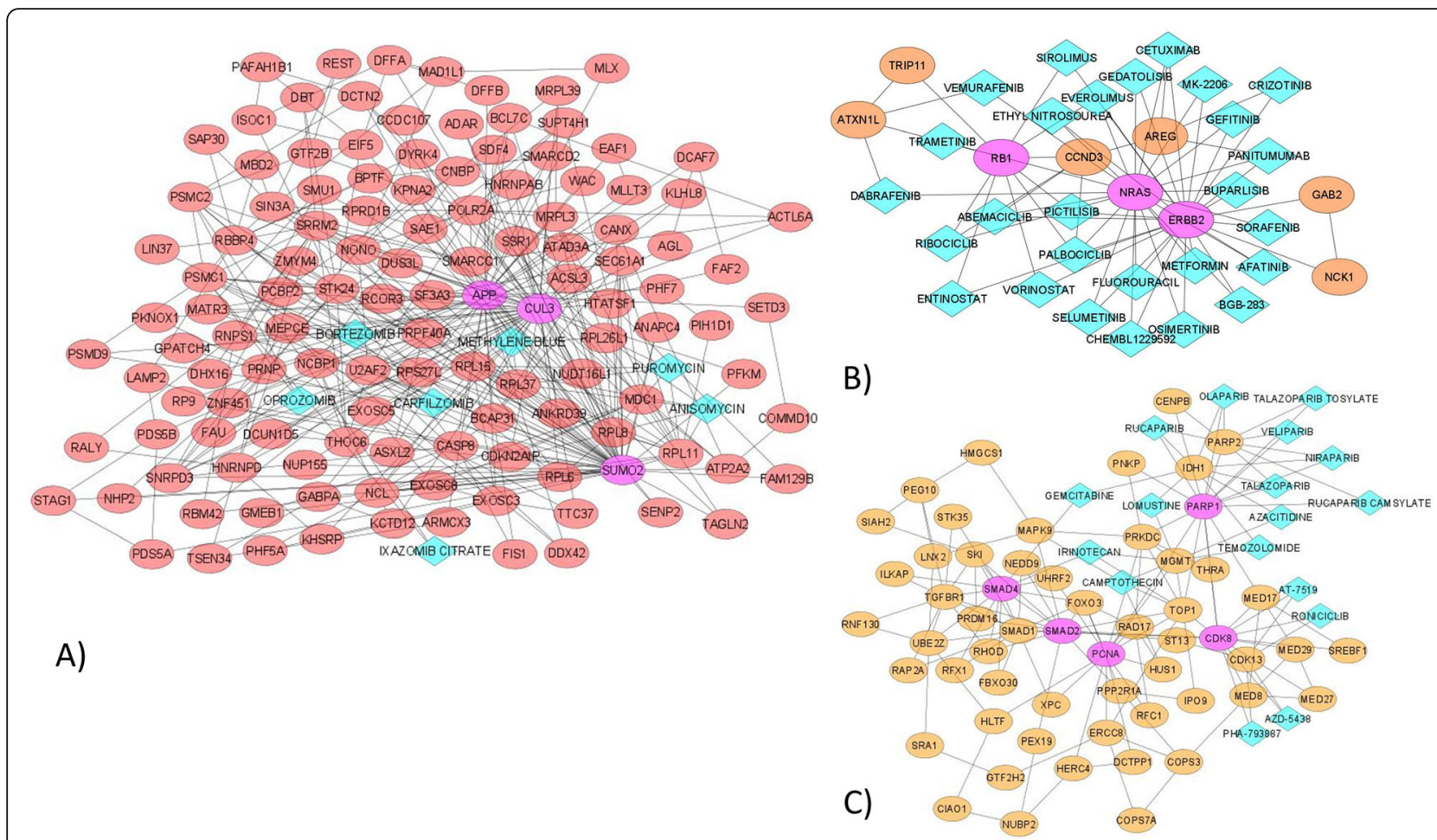

Fig. 3 Community detection. The figure shows the biggest communities identified a first community, $\mathbf{b}$ second community, $\mathbf{c}$ third community. Proteins with higher degree centrality are represented with the purple circles. Drugs are represented with light-blue triangle (we removed the nodes that have only one connection)

with the major number of drug targets in the network is palbociclib, and it interacts with 4 proteins: CCND3, ERBB2, NRAS and RB1 (Fig. 3b).

In the third community we found 5 genes with the highest d.c.: PARP1 (d.c. = 16), PCNA (d.c. = 14) SMAD4 (d.c. $=14)$, CDK8 (d.c. = 14) and SMAD2 (d.c. = 14). The drugs involved in this community are 17: veliparib, olaparib, rucaparib, niraparib, talazoparib, talazoparib tosylate, rucaparib camsylate, camptothecin, temozolomide, azacitidine, lomustine, gemcitabine, roniciclib, irinotecan, PHA-793887, AT-7519 and AZD-5438. The drug with the major number of drug targets in the network is camptothecin. It interacts with 4 proteins: MAPK9, HUS1, MGMT and TOP1 (Fig. 3c).

\section{Molecular docking}

Docking study was performed on 9 potential drugs (CHEMBL1161866, bortezomib, puromycin, metformin hydrochloride, NV-128, ME-344, methylene blue, anisomycin, and everolimus) against SARS-CoV-2.

The list of drugs examined for docking study is described in Table 1.

The binding energies of CHEMBL1161866 and ME344 with the main proteases of SAR-CoV-2 are presented in Supplementary 2. CHEMBL1161866 obtained a high binding energy showing a low affinity with the main proteases of SAR-CoV-2. Although ME-344 obtained a good performance, its possible role as drug against COVID-19 is not yet reported in literature.

Two drugs (Puromycin and anisomycin) obtained a negative binding energy value as shown in the Table 2 . Puromycin showed promising results in 4 out of 5 protein structures of SARS-CoV-2 with a binding energy lower than - 6.6. Anisomycin was found to interact with 2 out 5 protein structures of SARS-CoV-2 with a binding energy lower than -6 .

The details of the parameters for the considered conformations are presented in the Supplementary 2.

Docking interactions of puromycin with PDB ID 5R82 and anisomycin with PDB ID 5R7Z, which obtained the best binding energies, are presented in Fig. 4a-b.

Binding interactions of puromycin with PDB ID 5R82 involve 1 hydrophobic interaction and 4 hydrogen bonds (Fig. 4a). Purine group shows $\mathrm{H}$-bonding interactions with amino acid GLN127A, two hydroxyl groups interact with LYS5A and GLU288A and HN group interacts with GLU288A. Hydrophobic interaction is observed between carbon of methoxyphenyl group and GLU288A.

Binding interactions of anisomycin with PDB ID 5R7Z involve 6 hydrophobic interactions and 1 hydrogen bond (Fig. 4b). VAL202 forms hydrogen bond with 'O' of acetate group. Carbon of pyrrolidine interacts by forming 
Table 1 Chemical structure and mechanism of action of the most relevant drugs

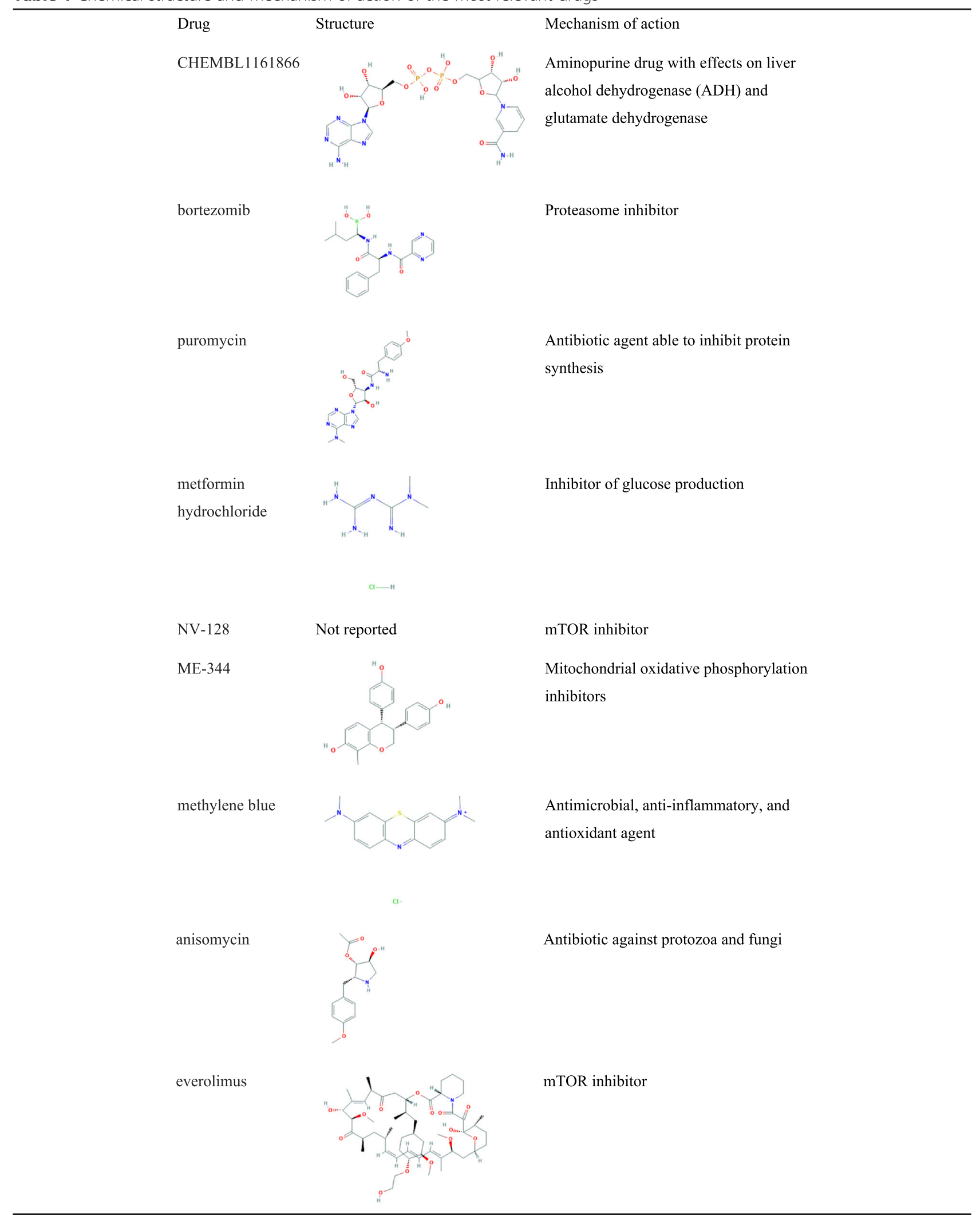


Table 2 Binding energy of two drugs identified in the docking study

\begin{tabular}{llllll}
\hline \multirow{2}{*}{ Ligand } & \multicolumn{6}{l}{ Binding Energy kcal/mol } \\
\cline { 2 - 6 } & $5 \mathrm{R} 7 \mathrm{Y}$ & $5 \mathrm{R} 7 \mathrm{C}$ & $5 \mathrm{R} 80$ & $5 \mathrm{R} 81$ & $5 \mathrm{R} 82$ \\
\hline puromycin & -6.78 & -6.81 & -4.98 & -6.65 & -6.94 \\
anisomycin & -5.47 & -6.41 & -5.44 & -5.6 & -6.08 \\
\hline
\end{tabular}

hydrophobic interactions with amino acid PRO132A, GLU240A and ILE200A. Carbon of acetate group interacts with GLU240A and carbon of methoxyphenyl interacts with VAL202A and ILE200A.

\section{Discussion}

Our in silico study highlighted protein interactions between genes differentially expressed in COVID-19 patients with respect to negative controls. Such proteins are involved in multiple complexes and biological processes including cell cycle, immune regulation, transcription and protein trafficking. Against these proteins, following in silico tests, we found several drugs, including FDA approved components, as potential treatments for COVID-19.

\section{Pathway analysis}

We found 22 pathways enriched with 1269 differentially expressed genes between lung biopsies from postmortem COVID-19 patients and lung biopsies from negative controls. Intriguing, the top differentially expressed mRNAs were found associated with Alzheimer's disease.

The risk of contracting COVID-19 in patients with Alzheimer disease (AD) is higher. This is due to their difficulty in following the recommendations from public health authorities and the difficulty of maintaining isolation during behavioural and psychological symptoms of dementia. In addition, patients with $\mathrm{AD}$ often have age and comorbid medical conditions associated with a poor prognosis and mortality from COVID-19 [19]. Few publications are currently available on a direct connection between the pathology of AD and COVID-19. From the little we know in the inflammatory response triggered by SARS-CoV-2, during this infection lymphopenia is observed, with loss of CD4 + and CD8 + T cells, hyperproduction of IL6, IL10, IL2R, TNFa and CCL2 [20]. The devastating effect of the cytokine storm on the respiratory system is already known. Less known is the effect of this inflammatory state on the nervous system. Chronic neuroinflammation associated with high levels of cytokine/chemokines has been associated with the pathophysiology of some neurodegenerative diseases (multiple sclerosis, Parkinson's disease, Alzheimer's disease, Alzheimer's disease, Huntington's disease or amyotrophic lateral sclerosis) [21]. In the case of $\mathrm{AD}$, it has been described that microglia cells lose the ability of degrading $\mathrm{A} \beta$ protein in the presence of proinflammatory cytokines (mainly IL1 or IL6), leading to the pathogenic deposits

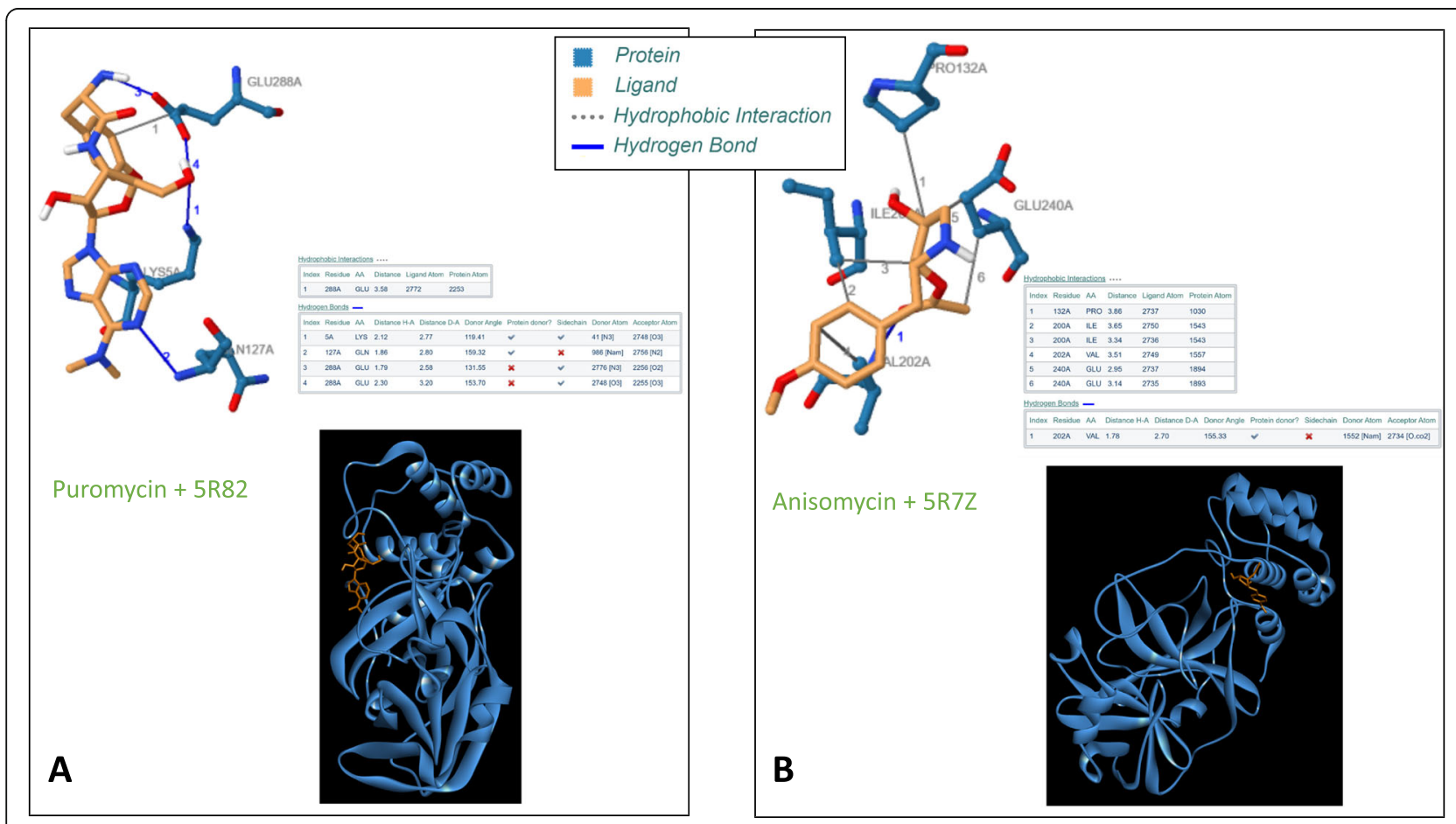

Fig. 4 Docking interactions of a puromycin with PDB ID 5R82 and $\mathbf{b}$ anisomycin with PDB ID 5R7Z 
of this protein in the brain [22]. It is thus possible that the cytokine storm produced by SARS-CoV-2 infection could worsen the status of AD patients.

Among the top differentially expressed mRNAs we also found mRNAs associated with human T-cell leukemia virus 1 infection, Shigellosis, hepatocellular carcinoma, viral carcinogenesis, apoptosis, non-alcoholic fatty liver disease (NAFLD), mTOR signaling, cellular senescence, protein processing in endoplasmic reticulum and cell cycle.

Chronic lymphocytic leukemia (CLL) is the most common adult leukemic disorder in the EuroAmerican population. It is caused by human retrovirus named human $\mathrm{T}$ lymphotropic viruses 1 (HTLV-1). HTLV-1 is very similar and relevant to human coronavirus (HCoVs) in animal-to-human transmission event. Although it is not yet fully known how SARS-CoV-2 interacts with host antiviral immunity, similar mechanisms are found from other $\mathrm{HCoVs}$ and human pathogenic viruses in other families that are very similar including HTLV-1 and the human immunodeficiency viruses (HIVs) [23]. Several therapeutic options are currently at hand for the first-line or relapsed/refractory CLL, including combination of drugs (i.e., bendamustine, alemtuzumab, ofatumumab) with corticosteroids. Corticosteroids are frequently used to treatment persons with these coronavirus infections in order to counteract high interleukin-6 concentrations, although some studies are still uncertain about the real efficacy and benefit of the use of corticosteroids in COVID-19 patients [24].

Virus infection alters the gut microbiome in humans and makes the organism more susceptible to intestinal bacterial infections such as Shigellosis. Shigellosis is an infectious intestinal disease, also called bacillary dysentery, caused by the genus Shigella spp. The expression of ACE2 is abundant in the epithelia of the lungs as well as in intestinal epithelial cells. In line with this scenario, COVID-19 may be associated with gut microbiota [25]. The connection between gastrointestinal tract and the respiratory tract, takes place through shared mucosal immune system which could justify the main symptoms of COVID-19: pneumonia and digestive infections [26, 27].

In addition to the lung and intestine, several evidences showed possible implications of hepatic involvement in SARS-Cov-2 infection. Patients with liver diseases, including hepatocellular carcinoma and Non-alcoholic fatty liver disease (NAFLD) (a metabolic disorder due to the accumulation of fat in the liver), can be more susceptible to the serious consequences of the COVID-19. This could be due to reduced innate immunity to the virus, as the liver plays crucial role in innate and adaptive immunity. As previously reported, the expression of ACE2 is abundant also in the epithelia of intestine, and as there is a rich circulation of blood from the intestine to liver, the spread of the virus in the liver is expected. Reduced hepatic innate immune status could contribute to progression of COVID-19 [28-30].

The mammalian target of rapamycin (mTOR) signaling pathway is a conserved serine/threonine kinase and promotes the cellular proliferation, protein synthesis and DNA replication. mTOR has been already associated with the development of influenza by promoting influenza virus replication [31]. The antiviral properties of mTORs inhibitors have been attributed to a variety of processes. Virus-infected cells initiate the stress response by promoting autophagy to destroy the infecting organism or by activating apoptosis to reduce virus spread. Since mTOR signaling pathway not only inhibits apoptosis but also reduces stress-activated autophagy, mTOR activation could have also a role in vaccine production to enhance virus replication [32, 33].

Apoptosis, cellular senescence, protein processing in endoplasmic reticulum and cell cycle are all processes that are altered as consequence of the virus infection. The infected cell promotes an activation of apoptosis and cell senescence, and an inhibition of protein synthesis and cell cycle in order to remove the infecting organism and reduce the spread of the virus.

\section{Antiviral proprieties of existing drugs targeting SARS- \\ CoV-2 genes}

We investigated the role of existing drugs in the protein network finding the drugs with the highest degree centrality in the network: CHEMBL1161866 (d.c. =11), bortezomib $($ d.c. $=6)$, puromycin (d.c. =6), metformin hydrochloride (d.c. =6), NV-128 (d.c.=6), ME-344 (d.c.=6), methylene blue (d.c. =5), anisomycin (d.c. = 5) and everolimus (d.c. = 5) (Fig. 2b).

CHEMBL1161866 is an aminopurine drug with effects on liver alcohol dehydrogenase $(\mathrm{ADH})$ and glutamate dehydrogenase, suggesting a possible beneficial application of this drug on liver infection by SARS-CoV-2. ADH enzymes, highly expressed in liver and colorectal tissues, catalyzed the conversion of alcohol to aldehydes during alcohol metabolism. In particular, $\mathrm{ADH}$ could have a role in hepatitis $B$ virus (HBV) and hepatitis $C$ virus infection, being the increased activity of ADH enzymes a clinical marker of liver injury [34]. Although a clear connection between COVID-19 and CHEMBL1161866 has been not proven yet, we could not exclude a possible beneficial effect of this drug on the COVID-19 patients.

Bortezomib (also known as velcade), a proteasome inhibitor approved against multiple myelomas, showed in several studies its antiviral proprieties. Proteasome inhibitors are encouraging antiviral agents that could 
inhibit viral entry. The ubiquitin-proteasome system (UPS) is involved in different steps of a broad range of viruses' replication cycle, including coronavirus. The UPS regulates many different processes, such as the cell cycle progression and apoptosis. In addition, ubiquitination is involved in the host immune response to viral infection regulating antigen presentation [35-37]. The Food and Drug Administration (FDA) has approved bortexomib to treat multiple myeloma.

Recent studies suggested that a drug already FDA approved for use against diabetes, metformin, may be effective against COVID-19. Metformin inhibits glucose production decreasing intestinal glucose absorption and enhancing glucose uptake by peripheral tissues. People with diabetes mellitus (DM) have poor prognosis and increased fatality in a wide range of viral infections, including SARS and MERS. The link between COVID-19 and DM could be explained by the use of angiotensin-converting enzyme inhibitors (ACEi) and angiotensin-receptor blockers (ARBs) in people with DM. Indeed, ACEi/ARBs act upregulating the receptor for entry of the virus into host pneumocytes. However, Metformin does not interfere with ACE2, and it is not clear how it could act on patients' outcome [38, 39]. To date, 20 clinical trials involve metformin as potential treatment of patients with virus diseases. These studies analyzed the effects of metformin in several viral infections such as Chronic Hepatitis C Infection (NCT02972723) and Human Immunodeficiency (NCT04500678).

Methylene blue is a fluorescent dye used for nucleic acid stain. It has been approved by the Food and Drug Administration (FDA) as treatment of methemoglobinemia. Due to its antimicrobial, antiinflammatory, and antioxidant effects, Methylene blue has been successfully utilized in vitro. Its antiviral proprieties have been attributed to its ability to intercalate into nucleic acids and inactivate RNA viruses. Virus inactivation with Methylene blue was applied in plasma to mitigate the risk of transmission by transfusion in human immunodeficiency virus (HIV), hepatitis $B$ virus, hepatitis $C$ virus and Zika virus [40, 41]. Methylene blue (also known as Provayblue) is indicated by FDA for the treatment of acquired methemoglobinemia. To date, only one on-going clinical trial (NCT04376788) involves Methylene blue as potential treatment of patients with COVID-19. Unfortunately, the results of this clinical study are not yet available.

Anisomycin was originally defined by FDA as an antibiotic against protozoa and fungi. The antiviral effect of anisomycin was demonstrated in cell cultures of Dengue and Zika viruses, mainly involving viral macromolecular synthesis [42]. In addition, anisomycin was reported to inhibit the animal picornavirus encephalomyocarditis virus [43] and to suppress in vitro replication of poliovirus [44] and flavivirus Japanese encephalitis virus [45].

Everolimus is an mTOR inhibitor with well-known antitumor activity in advanced cancer, including kidney and breast cancer. Everolimus showed also in vitro antiviral effects against influenza A virus: in a lethal mouse model of MERS, it delayed death and reduced MERS-CoV infection by $\sim 60 \%$. It has also been correlated with a reduced frequency of cytomegalovirus infection in transplant patients [46-48]. FDA approved everolimus for the treatment of neuroendocrine tumors, breast cancer and renal cell carcinoma. To date, 20 clinical trials involve everolimus as potential treatment of patients with viral infections. These studies analyzed the effects of everolimus in several virus diseases such as BK virus (NCT0162494 8) and hepatitis C virus (NCT01134952).

Puromycin is an antibiotic able to inhibit protein synthesis. Although protein inhibition could be obtained by puromycin treatment, in cornea $\mathrm{C}-\mathrm{M}$ virusinfected cells as well as in HeLa cells infected with Newcastle disease virus the viral particle continues to be produced due to viral protein intracellular accumulation. In this way, viral particles could be assembled, also in the absence of continuous protein synthesis [49]. It seems that puromycin could be effective only in the initial phases of a viral infection, when the viral protein is necessary to produce the viral particles for the initial virus spreading in the body. Although a clear connection between COVID19 and puromycin is not reported, we could not exclude a possible beneficial effect of this drug on the COVID-19 patients.

There is not also a clear connection between NV128, ME-344 and COVID-19, but we cannot exclude a future possible implication of these drugs in COVID-19 patients.

The docking studies estimated that two drugs (puromycin and anisomycin) have potential characteristics of binding to SARS-CoV-2.

\section{Conclusions}

This study identified, in silico, a group of proteins, drug targets of conventional treatments, within multiple complexes and biological processes that could represent effective mechanism of therapeutic actions also for SARS-CoV-2 infection. The protein interactions, that emerge from our analysis, highlight several drugs, as actionable molecules on proteins of the SARS-CoV-2-specific deregulated gene network; they could be proposed with a rational against the virus, alone or in combination with other therapies. 


\section{Supplementary information}

Supplementary information accompanies this paper at https://doi.org/10. 1186/s40360-020-00444-z.

Additional file 1: Supplementary 1. Differentially expressed genes between lung biopsies from postmortem COVID-19 positive patients and lung biopsies from negative controls. Supplementary 2. The details of the parameters for the conformations obtained with molecular docking analysis and the specific binding interactions

\section{Abbreviations}

ARDS: Acute respiratory distress syndrome; ACE2: Angiotensin-convertingenzyme-2; GEO: Gene Expression Omnibus; GTEx: Genotype-Tissue Expression; d.c.: Degree centrality; AD: Alzheimer disease; HCoVs: Human coronavirus; CLL: Chronic lymphocytic leukemia; HTLV-1: Human T lymphotropic viruses 1; mTOR: Mammalian target of rapamycin DM: Diabetes mellitus; ACEi: Angiotensin-converting enzyme inhibitors; ARBs: Angiotensin-receptor blockers

\section{Acknowledgements}

CNR thanks the generous support from Dassault Systèmes BIOVIA for the use of BIOVIA Discovery Studio Academic Research Suite in this work

\section{Authors' contributions}

CC has made substantial contribution to the acquisition, analysis and interpretation of data. GB has made substantial contribution to the interpretation of data. IC has revised the manuscript. The authors read and approved the final manuscript.

\section{Funding}

This work was supported by Italian Ministry of University and Research (MIUR) SYSBIONET-Italian ROADMAP ESFRI Infrastructures.

\section{Availability of data and materials}

The datasets supporting the conclusions of this article are available in the Gene Expression Omnibus repository (accession number: GSE147507) and Genotype-Tissue Expression (GTEx) project. The links of the datasets are: https://www.ncbi.nlm.nih.gov/geo/query/acc.cgi?acc=GSE147507 (2 lung bi opsies from postmortem COVID-19 positive male patients and 2 lung biopsies from COVID-19 negative male controls), https://gtexportal.org/home/ (gene expression levels of lung tissues from healthy subjects (320 healthy volunteers)). The crystal structures of SARS-CoV-2 main proteases (accession numbers PDB IDs: 5R7Y, 5R7Z, 5R80, 5R81 and 5R82) were obtained from Protein Data Bank (https://www.rcsb.org/).

\section{Ethics approval and consent to participate}

Not applicable.

\section{Consent for publication}

Not applicable.

\section{Competing interests}

The authors declare that they have no competing interests.

\section{Author details}

${ }^{1}$ Institute of Molecular Bioimaging and Physiology, National Research Council (IBFM-CNR), Via F. Cervi 93, 20090 Segrate-Milan, Milan, Italy. ${ }^{2}$ Department of Physics "Giuseppe Occhialini", University of Milan-Bicocca Piazza dell'Ateneo Nuovo, 1 - 20126 Milan, Italy.

Received: 15 May 2020 Accepted: 25 August 2020

Published online: 03 September 2020

\section{References}

1. Gordon DE, Jang GM, Bouhaddou M, Xu J, Obernier K, White KM, et al. A SARS-CoV-2 protein interaction map reveals targets for drug repurposing. Nature. 2020. https://doi.org/10.1038/s41586-020-2286-9.

2. Matricardi PM, Dal Negro RW, Nisini R. The first, holistic immunological model of COVID-19: Implications for prevention, diagnosis, and public health measures. Pediatr Allergy Immunol. 2020. https://doi.org/10.1111/pai. 13271.

3. Ge XY, Li JL, Yang XL, Chmura AA, Zhu G, Epstein JH, et al. Isolation and characterization of a bat SARS-like coronavirus that uses the ACE2 receptor. Nature. 2013;503(7477):535-8. https://doi.org/10.1038/nature12711.

4. Cava C, Bertoli G, Castiglioni I. In silico discovery of candidate drugs against Covid-19. Viruses. 2020;12(4). https://doi.org/10.3390/v12040404.

5. Jawhara S. Could intravenous immunoglobulin collected from recovered coronavirus patients protect against COVID-19 and strengthen the immune system of new patients? Int J Mol Sci. 2020;21(7). https://doi.org/10.3390/ ijms21072272.

6. Colaprico A, Silva TC, Olsen C, Garofano L, Cava C, Garolini D, et al. TCGAbiolinks: an R/bioconductor package for integrative analysis of TCGA data. Nucleic Acids Res. 2016;44(8):e71. https://doi.org/10.1093/nar/gkv1507.

7. Huber W, Carey VJ, Gentleman R, Anders S, Carlson M, Carvalho BS, et al. Orchestrating high-throughput genomic analysis with bioconductor. Nat Methods. 2015;12(2):115-21. https://doi.org/10.1038/nmeth.3252.

8. Yu G, Wang LG, Han Y, He QY. clusterProfiler: an R package for comparing biological themes among gene clusters. OMICS. 2012;16(5):284-7. https:// doi.org/10.1089/omi.2011.0118.

9. Cava C, Bertoli G, Castiglioni I. In silico identification of drug target pathways in breast cancer subtypes using pathway cross-talk inhibition. J Transl Med. 2018;16(1):154. https://doi.org/10.1186/s12967-018-1535-2.

10. Cava C, Pini S, Taramelli D, Castiglioni I. Perturbations of pathway coexpression network identify a core network in metastatic breast cancer. Comput Biol Chem. 2020;87:107313. https://doi.org/10.1016/j. compbiolchem.2020.107313.

11. Cava C, Colaprico A, Bertoli G, Graudenzi A, Silva TC, Olsen C, et al. SpidermiR: an R/Bioconductor package for integrative analysis with miRNA data. Int J Mol Sci. 2017;18(2). https://doi.org/10.3390/ijms18020274.

12. Wagner AH, Coffman AC, Ainscough BJ, Spies NC, Skidmore ZL, Campbell KM, et al. DGldb 2.0: mining clinically relevant drug-gene interactions. Nucleic Acids Res. 2016;44(D1):D1036-44. https://doi.org/10.1093/nar/ gkv1165.

13. Clauset A, Newman ME, Moore C. Finding community structure in very large networks. Phys Rev E Stat Nonlinear Soft Matter Phys. 2004;70 6 Pt 2 066111

14. Kim S, Chen J, Cheng T, Gindulyte A, He J, He S, et al. PubChem 2019 update: improved access to chemical data. Nucleic Acids Res. 2019;47(D1): D1102-9. https://doi.org/10.1093/nar/gky1033.

15. BDassault Systèmes BIOVIA, Discovery Studio Modeling Environment, Release; 2020

16. Rizvi SM, Shakil S, Haneef M. A simple click by click protocol to perform docking: AutoDock 4.2 made easy for non-bioinformaticians. EXCLI J. 2013;12:831-57.

17. Morris GM, Huey R, Olson AJ. Using AutoDock for ligand-receptor docking. Curr Protoc Bioinformatics. 2008Chapter 8:Unit 8.14. https://doi.org/10.1002/0471250953. bi0814s24.

18. Salentin S, Schreiber S, Haupt VJ, Adasme MF, Schroeder M. PLIP: fully automated protein-ligand interaction profiler. Nucleic Acids Res. 2015 Jul 1; 43(W1):W443-7. https://doi.org/10.1093/nar/gkv315.

19. Brown EE, Kumar S, Raiji TK, Pollock BG, Mulsant BH. Anticipating and mitigating the impact of the COVI-19 pandemic on Alzheimer's disease and related dementias [published online ahead of print, 2020 Apr 18]. Am J Geriatr Psychiatry. 2020:\$1064 7481(20)30294-3. https:/doi.org/10.1016/j.jagp.2020.04.010

20. Chen G, Wu D, Guo W, Cao Y, Huang D, Wang H, et al. Clinical and immunological features of severe and moderate coronavirus disease 2019. J Clin Invest. 2020;130(5):2620-9. https://doi.org/10.1172/JCl137244.

21. Frank-Cannon TC, Alto LT, McAlpine FE, Tansey MG. Does neuroinflammation fan the flame in neurodegenerative diseases? Mol Neurodegener. 2009;4:47. https://doi.org/10.1186/1750-1326-4-47.

22. Koenigsknecht-Talboo J, Landreth GE. Microglial phagocytosis induced by fibrillar beta-amyloid and lgGs are differentially regulated by proinflammatory cytokines. J Neurosci. 2005;25(36):8240-9.

23. Fung SY, Yuen KS, Ye ZW, Chan CP, Jin DY. A tug-of-war between severe acute respiratory syndrome coronavirus 2 and host antiviral defence: lessons from other pathogenic viruses. Emerg Microbes Infect. 2020;9(1):558-70. https://doi.org/10.1080/22221751.2020.

24. Li H, Chen C, Hu F, Wang J, Zhao Q Gale RP et al. Impact of corticosteroid therapy on outcomes of persons with SARS-CoV-2, SARS-CoV, or MERS-CoV infection: a systematic review and meta-analysis. Leukemia. 2020. https://doi. org/10.1038/s41375-020-0848-3 [Epub ahead of print]. 
25. Gao QY, Chen YX, Fang JY. 2019 novel coronavirus infection and gastrointestinal tract. J Dig Dis. 2020;21(3):125-6. https://doi.org/10.1111/ 1751-2980.12851.

26. Budden KF, Gellatly SL, Wood DL, Cooper MA, Morrison M, Hugenholtz P, et al. Emerging pathogenic links between microbiota and the gut-lung axis. Nat Rev Microbiol. 2017;15(1):55-63. https://doi.org/10.1038/nrmicro.2016.142.

27. He Y, Wen Q, Yao F, Xu D, Huang Y, Wang J. Gut-lung axis: the microbial contributions and clinical implications. Crit Rev Microbiol. 2017;43(1):81-95 https://doi.org/10.1080/1040841X.2016.1176988.

28. Prins GH, Olinga P. Potential implications of COVID-19 in non-alcoholic fattyliver disease. Liver Int. 2020. https://doi.org/10.1111/liv.14484 [Epub ahead of print].

29. Pirola CJ, Sookoian S. SARS-CoV-2 virus and liver expression of host receptors: putative mechanisms of liver involvement in COVID-19. Liver Int 2020. https://doi.org/10.1111/liv.14500 [Epub ahead of print].

30. Ji D, Qin E, Xu J, Zhang D, Cheng G, Wang Y, et al. Non-alcoholic fatty liver diseases in patients with COVID-19: a retrospective study. J Hepatol. 2020. https://doi.org/10.1016/j.jhep.2020.03.044 [Epub ahead of print]

31. Lehrer S. Inhaled biguanides and mTOR inhibition for influenza and coronavirus (review). World Acad Sci J. 2020;2(3). https://doi.org/10.3892/ wasj.2020.42.

32. Maiese K. The mechanistic target of rapamycin (mTOR): novel considerations as an antiviral treatment and possibilities for COVID-19. Curr Neurovasc Res. 2020 Apr 25. https://doi.org/10.2174/ 1567202617666200425205122

33. Bowman LJ, Brueckner AJ, Doligalski CT. The role of mTOR inhibitors in the Management of Viral Infections: a review of current literature. Transplantation. 2018;102(2S Suppl 1):S50-9. https://doi.org/10.1097/TP. 0000000000001777.

34. Jelski W, Strumnik A, Orywal K, Lapinski TW, Swiderska M, Szmitkowski M. Activity of alcohol dehydrogenase isoenzymes and aldehyde dehydrogenase in sera of patients with hepatitis C. Arch Med Sci. 2018; 14(2):281-7. https://doi.org/10.5114/aoms.2016.60406.

35. Al Saleh AS, Sher T, Gertz MA. Multiple myeloma in the time of COVID-19. Acta Haematol. 2020;17:1-7. https://doi.org/10.1159/000507690.

36. Schneider M, Ackermann K, Stuart M, Wex C, Protzer U, Schätzl HM, et al. Severe acute respiratory syndrome coronavirus replication is severely impaired by MG132 due to proteasome-independent inhibition of Mcalpain. J Virol. 2012;86(18):10112-22. https://doi.org/10.1128/JVI.01001-12.

37. Raaben M, Posthuma CC, Verheije MH, te Lintelo EG, Kikkert M, Drijfhout JW, et al. The ubiquitin-proteasome system plays an important role during various stages of the coronavirus infection cycle. J Virol. 2010;84(15):786979. https://doi.org/10.1128/JVI.00485-10.

38. Pal R, Bhadada SK. Should anti-diabetic medications be reconsidered amid COVID-19 pandemic? Diabetes Res Clin Pract. 2020;163:108146. https://doi. org/10.1016/j.diabres.2020.108146.

39. Nakhleh A, Shehadeh N. Interactions between antihyperglycemic drugs and the renin-angiotensin system: putative roles in COVID-19. A mini-review. Diabetes Metab Syndr. 2020;14(4):509-12. https://doi.org/10.1016/j.dsx.2020. 04.040 .

40. Nedu ME, Tertis M, Cristea C, Georgescu AV. Comparative study regarding the properties of methylene blue and Proflavine and their optimal concentrations for in vitro and in vivo applications. Diagnostics (Basel). 2020 10(4). https://doi.org/10.3390/diagnostics10040223.

41. Wang Y, Ren K, Liao X, Luo G, Kumthip K, Leetrakool N, et al. Inactivation of Zika virus in plasma and derivatives by four different methods. J Med Virol. 2019;91(12):2059-65. https://doi.org/10.1002/jmv.25538.

42. Quintana VM, Selisko B, Brunetti JE, Eydoux C, Guillemot JC, Canard B, et al. Antiviral activity of the natural alkaloid anisomycin against dengue and Zika viruses. Antiviral Res. 2020;176:104749. https://doi.org/10.1016/j.antiviral.2020. 104749

43. Ramabhadran TV, Thach RE. Specificity of protein synthesis inhibitors in the inhibition of encephalomyocarditis virus replication. J Virol. 1980;34(1):293-6.

44. Hwang YC, Chu JJ, Yang PL, Chen W, Yates MV. Rapid identification of inhibitors that interfere with poliovirus replication using a cell-based assay. Antiviral Res. 2008;77(3):232-6. https://doi.org/10.1016/j.antiviral.2007.12.009.

45. Chang CC, Ou YC, Raung SL, Chen CJ. Antiviral effect of dehydroepiandrosterone on Japanese encephalitis virus infection. J Gen Virol. 2005;86(Pt9):2513-23. https://doi.org/10.1099/vir.0.81123-0.

46. Murray JL, McDonald NJ, Sheng J, Shaw MW, Hodge TW, Rubin DH, et al. Inhibition of influenza a virus replication by antagonism of a PI3K-AKT-
mTOR pathway member identified by gene-trap insertional mutagenesis. Antivir Chem Chemother. 2012;22(5):205-15. https://doi.org/10.3851/ IMP2080.

47. Dyall J, Gross R, Kindrachuk J, Johnson RF, Olinger GG Jr, Hensley LE, et al. Middle East respiratory syndrome and severe acute respiratory syndrome: current therapeutic options and potential targets for novel therapies. Drugs. 2017;77(18):1935-66. https://doi.org/10.1007/s40265-017-0830-1.

48. Kindrachuk J, Ork B, Hart BJ, Mazur S, Holbrook MR, Frieman MB, et al. Antiviral potential of ERK/MAPK and PI3K/AKT/mTOR signaling modulation for Middle East respiratory syndrome coronavirus infection as identified by temporal kinome analysis. Antimicrob Agents Chemother. 2015;59(2):108899. https://doi.org/10.1128/AAC.03659-14.

49. Northrop RL. Effect of puromycin and actinomycin D on a persistent mumps virus infection in vitro. J Virol. 1969;4(2):133-40.

\section{Publisher's Note}

Springer Nature remains neutral with regard to jurisdictional claims in published maps and institutional affiliations.
Ready to submit your research? Choose BMC and benefit from:

- fast, convenient online submission

- thorough peer review by experienced researchers in your field

- rapid publication on acceptance

- support for research data, including large and complex data types

- gold Open Access which fosters wider collaboration and increased citations

- maximum visibility for your research: over $100 \mathrm{M}$ website views per year

At BMC, research is always in progress.

Learn more biomedcentral.com/submissions 